\title{
Linx
}

Revue des linguistes de l'université Paris X Nanterre

$82 \mid 2021$

Entre vieillissement et innovation : le changement linguistique

\section{Changement, innovation et disparition dans les systèmes grammaticaux : l'exemple de la classification nominale}

Change, innovation, lost in the grammatical system: the example of the nominal classification

\section{Alice Vittrant et Sylvie Voisin}

\section{(2) OpenEdition}

\section{Journals}

Édition électronique

URL : https://journals.openedition.org/linx/8080

DOI : $10.4000 / \operatorname{linx} .8080$

ISSN : 2118-9692

\section{Éditeur}

Presses universitaires de Paris Nanterre

Référence électronique

Alice Vittrant et Sylvie Voisin, « Changement, innovation et disparition dans les systèmes grammaticaux:

l'exemple de la classification nominale », Linx [En ligne], 82 | 2021, mis en ligne le 15 juillet 2021, consulté le 20 juillet 2021. URL : http://journals.openedition.org/linx/8080 ; DOI : https://doi.org/ $10.4000 /$ linx.8080

Ce document a été généré automatiquement le 20 juillet 2021.

Département de Sciences du langage, Université Paris Ouest 


\title{
Changement, innovation et disparition dans les systèmes grammaticaux : l'exemple de la classification nominale
}

Change, innovation, lost in the grammatical system: the example of the nominal classification

\author{
Alice Vittrant et Sylvie Voisin
}

\section{Introduction}

L'étude des changements linguistiques est une constante dans la linguistique depuis ses débuts notamment dans les travaux en linguistique historique en s'appuyant sur des comparaisons lexicales. Il s'agit de proposer des regroupements de langues en familles, basés sur des processus de changements réguliers, obéissant à des règles essentiellement phonologiques, telles que les lois de Grimm ou de Verner, par exemple. La régularité et les processus de changements qui constituent le fondement de la linguistique historique ont servi de base par la suite à des linguistes comme Meillet (1912) et plus récemment Heine (1984, 1993, 2003), Marchello-Nizia (1999), Peyraube (2002) inter al. Leurs travaux sur la grammaticalisation montrent les différentes évolutions possibles pour des items lexicaux et grammaticaux. Ils identifient différents mécanismes à l'origine de ces changements (disparition, recatégorisation, réanalyse, extension analogique, etc.) et une variété de facteurs susceptibles de les déclencher (Heine et Kuteva 2005, 2008, 2010; Marchello-Nizia 2006: 67-72). Participant de cette nouvelle approche du changement linguistique, les travaux sur le contact de langues (Thomason et Kaufman, 1988) vont ainsi mettre l'accent sur la place importante de l'emprunt dans l'évolution des langues, y compris dans la «création" d'items 
grammaticaux. En pointant des causes externes autant qu'internes, dans une approche dite 'de la grammaticalisation', ces différents travaux posent que c'est dans l'usage même de la langue que s'initie le changement. Heine (1997) montre ainsi que l'émergence de nouvelles formes grammaticales est corrélée à des schémas cognitifs sous-jacents. Il suggère donc une approche diachronique pour l'analyse des structures contenant ces formes émergentes, des procédés de grammaticalisation qui sont des voies universelles de création des items grammaticaux. Ce que l'on peut illustrer par la citation de Givón (1971: 413) « Today's morphology is yesterday's syntax ».

Ce renouveau des analyses sur le changement linguistique montre que items lexicaux et items grammaticaux peuvent relever des mêmes processus et être parfois directement influencés par des facteurs sociaux. Il montre aussi que l'émergence de nouvelles unités grammaticales peut être aussi rapide que pour les items lexicaux comme nous le verrons dans le présent article.

Il est assez commun que de nouveaux lexèmes voient le jour suite à la création d'un nouvel objet ou d'un nouveau concept, que cette innovation passe par une étape d'emprunt dans laquelle le terme s'adapte plus ou moins fortement à la langue emprunteuse ou que cette innovation lexicale soit le résultat d'un néologisme ou d'un glissement sémantique. Il est assez courant également que malgré son adaptation au système et les changements linguistiques que cette intégration implique, le terme tombe plus ou moins vite en désuétude. Ce «cycle de vie " pour les items lexicaux est difficilement prédictible puisqu'il dépend entièrement de facteurs sociaux et ne dépend que très rarement de facteurs linguistiques internes. Moins dépendants de facteurs sociaux, on pourrait supposer que les items grammaticaux qui participent du système et établissent un certain équilibre dans ce système (de Saussure 1916; Tuite 1999) aient un "cycle de vie» plus lent aussi bien au niveau de leur création, que de leur changement et de leur disparition.

Dans cet article, nous souhaitons montrer la similarité des processus d'émergence ou "cycles de vie" des items lexicaux et grammaticaux. Nous examinerons dans un premier temps les changements observables dans le lexique, que ces changements soient dus à la disparition d'une forme ou d'une fonction, ou à une innovation par apparition ou réanalyse d'une forme. Dans un deuxième temps, nous montrerons que les mêmes mécanismes de changement se retrouvent dans le domaine grammatical. Ces transformations, sans pleinement modifier le système, impliquent des réajustements qui peuvent passer relativement inaperçus, mais n'en affectent pas moins profondément le système. Nous illustrerons les modifications qui affectent les outils grammaticaux au moyen des marqueurs de classification nominale. Cette catégorisation grammaticale d'éléments nominaux recouvre plusieurs systèmes analysés comme plus ou grammaticalisés. Nous montrerons que dans les systèmes les plus grammaticalisés tels que les systèmes de genres des langues Niger-Congo ou dans les systèmes moins grammaticalisés tels que les systèmes de classificateurs des langues d'Asie, les innovations, changements, disparitions et modifications sont réguliers et parfois assez rapides, qu'ils répondent à des mécanismes d'innovation et d'emprunt. Nous posons que ce qui est valable pour le lexique, l'est également pour les éléments grammaticaux.

Notre article accréditera l'idée, déjà actée par les comparatistes (Boltanski 1998), que les changements linguistiques identifiés pour le lexique, le sont pour l'ensemble des 
éléments qui composent le système d'une langue, des sons aux lexèmes en passant par les morphèmes grammaticaux.

\section{Les items lexicaux}

Les changements rencontrés dans l'évolution du lexique d'une langue sont variés, et peuvent aboutir à la disparition d'une forme parce que l'élément qu'il désigne a disparu ou parce qu'un nouveau terme est utilisé pour le désigner. Dans ce dernier cas, l'item lexical n'est pas nécessairement voué à la disparition, il peut être utilisé pour désigner autre chose (réanalyse) donnant lieu parfois à l'émergence d'un nouveau sens. Les innovations lexicales peuvent aussi être liées à l'émergence d'un nouvel item. Sans prétendre à l'exhaustivité des situations, voici quelques exemples illustrant la variété de ces changements.

\subsection{Disparition de la forme ou du sens premier}

Pour les langues écrites et outillées comme le français, la réédition régulière de dictionnaires facilite l'étude des changements lexicaux. Ces rééditions s'accompagnent généralement de la disparition de termes auparavant attestés comme l'anglicisme « computeur " (1), tandis que d'autres items lexicaux voient leur signification évoluer comme dans le cas de 'chef', avec une perte du sens originel (2).

\begin{tabular}{|l|l|}
\hline (1) & $\begin{array}{l}\text { disparition de la forme linguistique computeur mais pas de l'objet : disparition du mot d'origine } \\
\text { anglaise au profit de la forme 'ordinateur' (disparition du dictionnaire Petit Larousse en 19981) }\end{array}$ \\
\hline $\begin{array}{l}\text { «Oudon obéit, emporta les documents. Figueras déphasait le computeur, regardait les voyants s'éteindre } \\
\text { l'un après l'autre. » (Laforest 1972, 175) }\end{array}$
\end{tabular}

(2) llissement sémantique du mot " chef » dont le sens «tête, chevelure » présent en Moyen
Français [jusqu'en 1694], a disparu pour prendre le sens de 'celui qui est à la tête d'un corps,
d'une assemblée, etc., qui y a le premier rang et la principale autorité.' [1935]²

On trouve de nombreux exemples de glissement sémantique par exemple dans l'ouvrage d'Alain Rey (2013). Le mot nuque désignait au départ «le centre nerveux, bulbe rachidien..." qui suite au progrès en anatomie, a été utilisé pour désigner l'arrière du crâne, rôle jusque-là attribué à chignon qui est resté dans l'usage, mais pour désigner une coiffure. Ce 'jeu des chaises musicales' décrit par (Rey, 2013 : 111) ressemble au 'principe du domino' qui permet d'expliquer les changements en chaîne que l'on est parfois amené à décrire en linguistique comparée pour les systèmes phonologiques.

«On observe dans des langues assez variées que des lacunes apparues dans les systèmes phonologiques ( $\mathrm{du}$ fait de la transformation d'un son en un autre) se trouvent comblées grâce à de nouveaux changements. Le même tableau des correspondances Vanuatu Nord montre qu'en Lemerig, après le changement ${ }^{*} \mathrm{k}>\mathrm{\gamma}$, c'est-à-dire après la disparition $d u^{*} \mathrm{k}$, un nouveau changement ${ }^{*} \mathrm{~g} g \mathrm{k}$ est intervenu. C'est sur ce type de changements qu'est bâtie la loi d'anthologie de Grimm qui rend compte des changements intervenus du proto-indo-européen au proto-germanique par une chaîne de transformations comme ${ }^{*} \mathrm{k}>\mathrm{h},{ }^{*} \mathrm{~g}>\mathrm{k}$, ${ }^{*} \mathrm{gh}>\mathrm{g}$. 
Ce type d'enchaînement n'a rien de mystérieux. La disparition du k fait perdre au trait de voisement sa fonction distinctive, et ainsi g passe à k. Par enchaînement, le changement du g prive de sa fonction le trait supplémentaire (l'aspiration) de gh, qui disparaît également. Parmi ce type de changements en chaîne, le plus long est à ma connaissance celui qui est observé en Basari et en Bedik (branche Nord de la famille atlantique) avec l'enchaînement des 5 changements suivants : *s $>$ š, r > s, *l $>\mathrm{r},{ }^{*} \mathrm{~d}>\mathrm{l},{ }^{*} \mathrm{n}>\mathrm{d}$. » (Pozdniakov, $\left.2016: 7\right)$

\subsection{Innovation via l'émergence d'un nouvel item ou réanalyse}

Les innovations faisant intervenir un nouvel item lexical sont courantes. Elles sont liées à l'émergence dans notre quotidien de nouveaux outils (wifi), de nouvelles pratiques (selfie), ou encore de modes, comme la mode culinaire. Comme l'illustrent les items lexicaux en (3) qui émergent dans nos usages linguistiques via de nouveaux aliments ou de nouvelles pratiques alimentaires.

\begin{tabular}{|l|l|l|l|}
\hline (3) & a. & smoothie & \\
\hline & b. & vegan & \\
\hline & c. & flexitarian & \\
\hline & d. & phô [fø] & soupe d'origine vietnamienne \\
\hline & e. & pokebowl [pokebol] & entrée d'origine hawaienne (cubes de poissons marinés) \\
\hline
\end{tabular}

Les cas de réanalyse sont plus subtils et moins directement perceptibles par les locuteurs. On pourrait citer la recatégorisation de l'adjectif «grave » (Yaguello, 1998: 30-34), du quantifieur «tarpin » du français méridional, tous deux ayant aujourd'hui acquis une fonction adverbiale. Autre cas de glissement sémantique intéressant celui des mots désignant "serviteur » et " seigneur » qui dans un grand nombre de langues d'Asie sont devenus des pronoms de première et deuxième personnes (Vittrant, 2019 : 84). Ces cas de réanalyse sont à la frontière lexique - grammaire et bien connu des syntacticiens. Ils soulèvent l'épineuse question de la catégorisation grammaticale, comme en atteste les titres de l'article de Feuillet (1983) «Se débarrassera-t-on un jour des parties du discours? ? ou de Anward et al. (1997) « Parts of speech: A challenge for typology " et du traitement que l'on doit faire de cette polyfonctionalité dans les langues (Robert, 2005).

Arrêtons-nous quelques instants sur tarpin, terme marseillais couramment utilisé par les jeunes qui appartient aujourd'hui à deux catégories grammaticales suite à une réanalyse. Utilisé comme quantifieur apportant une indication de pluralité à un nom (a) sur le modèle de beaucoup, il peut apparaître sans le nom qu'il détermine, lequel est repris par un pronom (b). Cette utilisation semble avoir favorisé sa réanalyse comme adverbe à fonction d'intensification apparaissant alors avec un adjectif comme en (c), les différents usages partageant une sémantique commune, la quantification. Réanalysé comme adverbe, tarpin peut alors étendre ses usages adverbiaux et modifier outre des adjectifs, des verbes, comme en (d). 


\begin{tabular}{|l|l|l|}
\hline (4) & a. & Marius a tarpin de chance ! \\
\hline & b. & Des pokemons, j'en ai tarpin \\
\hline & c. & Marius, il est tarpin beau ! \\
\hline & d. & Ce soir, j'ai tarpin joué à Fortnight ! \\
\hline
\end{tabular}

Comme le montre la carte ci-dessous, la réanalyse de ces termes (grave et tarpin) qui résultent d'innovations régionales, suivent les mêmes processus de réanalyse même à des périodes distinctes.

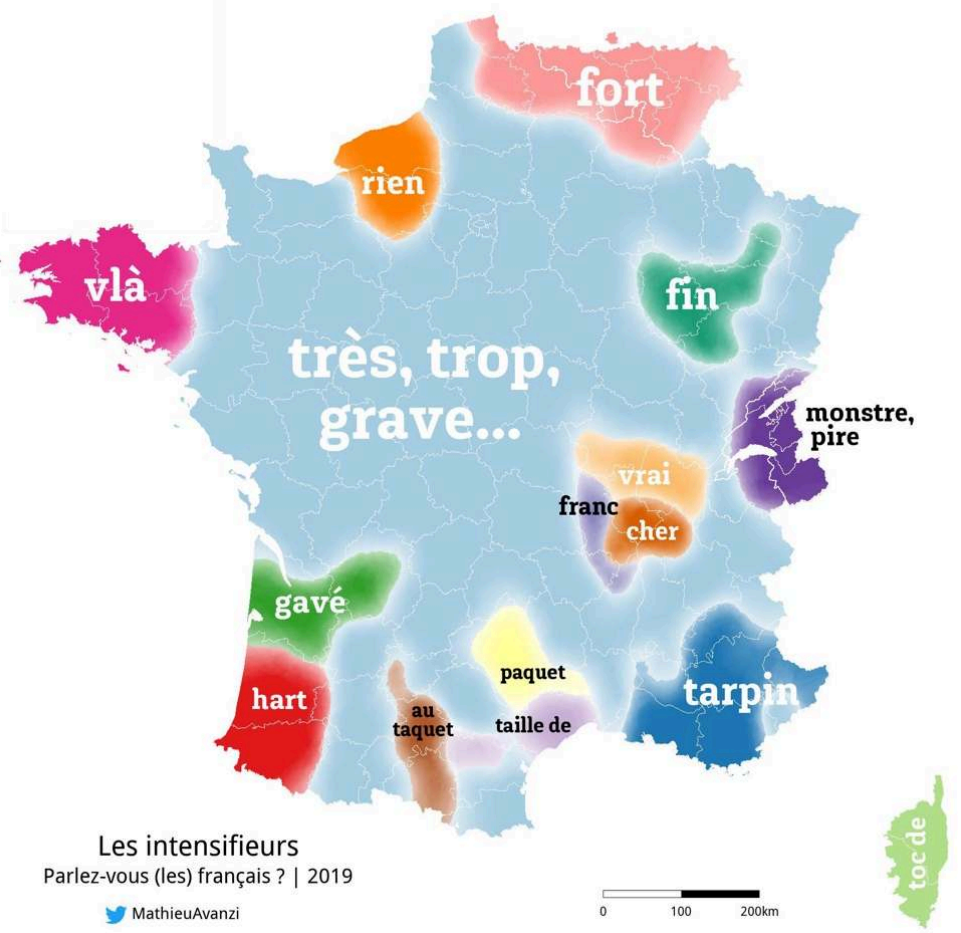

Autres exemples d'innovations par glissement sémantique et réanalyse, celui des pronoms de première et deuxième personnes en thaï, birman et dans d'autres langues du Sud-est asiatique. Ils sont issus de noms communs signifiant respectivement « serviteur» ou « seigneur» (Vittrant, 2019). Parfois, les pronoms sont issus d'autres noms communs, comme en thaï 0 , qui utilise le nom «souris» pour la forme humilifique de la $1^{\text {ère }}$ personne.

Tableau 1. Origine nominale des pronoms de $1^{\text {ère }}$ et $2^{\mathrm{e}}$ personnes en Asie du Sud-Est d'après Vittrant (2019)

\begin{tabular}{|l|l|l|l|ll|}
\hline LANGUE & FORME & PERS. & ORIGINE & DEGRÉ DE POLITESSE \\
\hline Thaï & /khâa'/ & $\begin{array}{l}1^{\text {ère }} \\
\text { pers. }\end{array}$ & serviteur, esclave & $\begin{array}{l}\text { pour s'adresser à des } \\
\text { inférieurs }\end{array}$ \\
\hline
\end{tabular}




\begin{tabular}{|c|c|c|c|c|}
\hline & /khâphacâw/ & $\begin{array}{l}1^{\text {ère }} \\
\text { pers. }\end{array}$ & $\begin{array}{l}\text { serviteur du chef/ } \\
\text { seigneur }\end{array}$ & terme formel \\
\hline & /câw'/ & $2^{\mathrm{e}}$ pers. & roi, seigneur & terme littéraire \\
\hline & /naaj'/ & $2^{\mathrm{e}}$ pers. & maître & $\begin{array}{l}\text { terme amical utilisé entre } \\
\text { pairs }\end{array}$ \\
\hline \multirow[t]{2}{*}{ Khmer } & 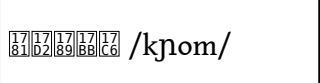 & $\begin{array}{l}1^{\text {ère }} \\
\text { pers. }\end{array}$ & esclave, serviteur & formel, poli \\
\hline & [17] & $2^{\mathrm{e}}$ pers. & $\begin{array}{l}<\text { seigneur, maître } \\
\text { (malais) }\end{array}$ & \\
\hline \multirow[t]{2}{*}{ Birman } & $\begin{array}{ll}10 \\
1007\end{array}$ & $\begin{array}{l}1^{\text {ère }} \\
\text { pers. }\end{array}$ & sujet (du roi), serviteur & $\begin{array}{l}\text { entre pairs, relativement } \\
\text { poli }\end{array}$ \\
\hline & 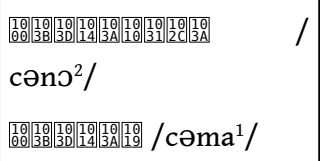 & $\begin{array}{l}1^{\text {ère }} \\
\text { pers. }\end{array}$ & $\begin{array}{l}\text { sujet du roi, serviteur / } \\
\text { sujet du roi (féminin) }\end{array}$ & neutre, poli \\
\hline & 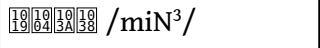 & $2^{\mathrm{e}}$ pers. & roi & familier \\
\hline & 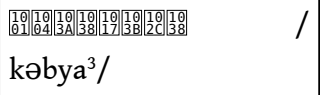 & $2^{\mathrm{e}}$ pers. & seigneur, maître & poli, utilisé par les hommes \\
\hline & 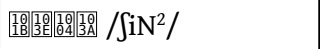 & $2^{\mathrm{e}}$ pers. & seigneur, maître & poli, utilisé par les femmes \\
\hline
\end{tabular}

Thai (d'après Iwasaki \& Ingkaphirom, 2005: 60)

\begin{tabular}{|l|l|l|l|l|}
\hline (5) & \multicolumn{4}{|l|}{ หนู ไม่ มี กาว } \\
\hline & nǔ: & mâj & mi: & ka:w \\
\hline & souris & NEG & avoir & colle \\
\hline & \multicolumn{4}{|l|}{ 'Je n'ai pas de colle.' } \\
\hline
\end{tabular}

À la suite de cette présentation de mécanismes de changements liés aux items lexicaux (disparition, réanalyse, extension ou glissement sémantique), nous examinons les mécanismes de changements que l'on rencontre dans le domaine grammatical.

\section{Les items grammaticaux : systèmes de classification nominale et changements}

Dans cette deuxième partie, nous ferons le parallèle entre les changements lexicaux évoqués ci-dessus et ceux décrits pour les items grammaticaux dans un domaine particulier, celui de la classification nominale. Notre démonstration s'appuiera 
essentiellement sur des données issues de nos terrains respectifs sur des langues d'Asie et d'Afrique.

Les deux systèmes de classification nominale qui seront abordés dans cette section ont la particularité d'être aux extrêmes d'une échelle de grammaticalité (Fedden et Corbett, 2018). Les classificateurs, après les termes de mesure, constituent un système lexicogrammatical de classification des noms qui reste assez motivé (Vittrant et Mouton, à paraître ; Grinevald,1999; Vittrant, 2005). À l'inverse, les classes nominales, avec le genre, sont des systèmes beaucoup plus arbitraires et considérés comme fortement grammaticalisés. Ils sont largement présents dans les langues Niger-Congo (Afrique) (Welmers, 1973; Creissels, 1991; Creissels et Pozdniakov, 2015).

Il est ainsi possible de montrer que dans les deux types de systèmes :

(1) les morphèmes grammaticaux peuvent changer de fonction ou de valeur, que ce constat résulte d'une comparaison avec d'autres langues (apparentées) ou d'une perte des propriétés attendues.

(2) les morphèmes grammaticaux peuvent s'étioler ou disparaitre complètement, soit que la forme ait disparu (Downing, 1996; Diagne, 2015), soit qu'elle ait été ré-analysée, recatégorisée.

(3) les morphèmes grammaticaux peuvent encore être le résultat d'innovations récentes.

On retrouve ainsi, les différents mécanismes de changements illustrés dans la section précédente pour les items lexicaux.

\subsection{Classification nominale et changement de fonction}

Avant d'entrer dans le détail de changements attestés dans le système de classification nominale qui vont nous permettre d'illustrer des phénomènes de changements identiques rencontrés dans le lexique, il convient de présenter rapidement la classification nominale et plus précisément les deux sous-systèmes qui serviront d'illustration ici, à savoir, le système des classificateurs et le système des classes nominales.

L'étude de la classification nominale dans les langues du monde renvoie d'une part à l'observation de la structure à travers laquelle les langues classent et organisent leur lexique nominal pour l'essentiel, et d'autre part aux fonctions grammaticales que remplissent ces structures. En tenant compte de ces différentes structures et fonctions, on a longtemps réparti les langues en trois types selon qu'elles organisent leur lexique à l'aide :

- du genre, à la façon de langues telles que le latin, le français, l'anglais ou l'hausa,

- des classes nominales, à la façon des langues bantoues, telles que le swahili, par exemple,

- des classificateurs, à la façon de nombre de langues d'Asie, entre autres.

Chacun de ces sous-systèmes a des particularités structurelles et fonctionnelles qui permettent de les distinguer; ils remplissent des fonctions différentes qui peuvent parfois se recouper.

Dans un système à classificateurs, les morphèmes ne catégorisent les noms que dans certains contextes, principalement dans les contextes de quantification (présence de 
numéraux, de quantifieurs) et de spécification (présence de démonstratifs, marqueur de définitude ou d'indéfinitude) (Vittrant et Tang, à paraître). À la différence des systèmes de genre, ils n'impliquent pas de phénomène d'accord, et sont considérés comme des systèmes semi-grammaticaux sur le continuum allant des systèmes lexicaux aux systèmes grammaticaux (Grinevald, $2015: 812$ ).

Le rôle du classificateur est d'intégrer sous une même étiquette, comme participant du même concept, une ou plusieurs entités. Les traits sémantiques encodés par les classificateurs relèvent de domaines sémantiques récurrents à différentes langues, à savoir (i) la forme ou configuration spatiale, (ii) la matière ou l'animacité, (iii) la consistance (flexible, mou, masse...), (iv) la taille, (v) la localisation, (vi) l'arrangement (type de groupements) et (vii) des quanta (unité de comptage comme la dizaine) (Vittrant et Mouton, à paraître).

Contrairement aux systèmes de genre, un nom peut être utilisé (et classé) à l'aide de différents classificateurs, selon la propriété sémantique que l'on veut mettre en avant.

Dans l'exemple (6) apparait le classificateur $/ \mathrm{si}^{3} /$, qui regroupe dans une même classe les voitures (a), les vélos (b) mais aussi les éléphants (c), trois entités qui ont en commun la caractéristique d'être un 'moyen de transport'. Les éléphants peuvent cependant aussi être catégorisés comme appartenant à la classe des animaux, et apparaitre lors d'une quantification avec le classificateur $/ \mathrm{kaJN}^{2} /$, soulignant ce trait inhérent.

Birman

\begin{tabular}{|c|c|c|c|c|c|c|c|c|}
\hline \multirow[t]{2}{*}{ (6) } & a. & \multicolumn{3}{|c|}{ 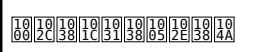 } & b. & \multicolumn{3}{|c|}{ 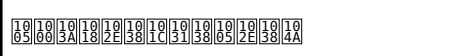 } \\
\hline & & $\mathrm{ka}^{3}$ & $1 e^{3}$ & $\mathbf{s i}^{3}$ & & səbriN & $1 e^{3}$ & $\mathbf{s i}^{3}$ \\
\hline & & voitures & 4 & & 1.transport & vélos & 4 & CLF: moyen.transport \\
\hline & & \multicolumn{4}{|c|}{ Quatre voitures } & \multicolumn{3}{|c|}{ Quatre vélos } \\
\hline
\end{tabular}

\begin{tabular}{|c|c|c|c|c|c|c|c|}
\hline \multirow[t]{2}{*}{ c. } & \multicolumn{3}{|c|}{ 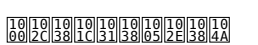 } & d. & \multicolumn{3}{|c|}{ 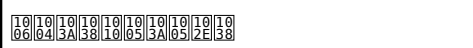 } \\
\hline & $\mathrm{ka}^{3}$ & to & $\mathbf{s i}^{3}$ & & $\operatorname{shiN}^{3}$ & to & $\mathbf{s i}^{3}$ \\
\hline & éléphant & 1 & & animal & éléphant & 1 & CLF: moyen.transport \\
\hline & \multicolumn{4}{|c|}{ un éléphant (animal) } & \multicolumn{3}{|c|}{ un éléphant (moyen de transport) } \\
\hline
\end{tabular}

En d'autres termes, la raison d'être des classificateurs peut être décrite ainsi : dans les langues possédant ce type de système, le statut conceptuel de tous les noms de la langue s'apparente à celui des noms de masse, des entités non-discrètes (essence, substance) auxquelles il faut donner une structure interne (délimitation) afin de pouvoir les associer, les compter avec des unités de même type. Cette délimitation se fait à l'aide du classificateur qui, en catégorisant l'entité par l'une de ses propriétés intrinsèques, en fait un individu qu'il est possible de compter à la manière des noms massifs. 
Dans le système des classes nominales, considéré depuis Corbett (1991) comme un soussystème du genre basé sur l'échelle de l'animacité, les marques de classes catégorisent les noms du lexique et servent à les dénombrer. Dans les langues à classes nominales, il y a généralement plus de 5 classes différentes. La classe d'un nom s'identifie par les accords qu'elle déclenche. Cet accord est, généralement dans les langues à classes, en interaction avec un préfixe nominal qui marque le nombre sur le nom, composant des systèmes de nombre à deux valeurs marquées; le singulier et le pluriel ont des morphèmes spécifiques et obligatoires. Les morphèmes d'accord ne sont pas nécessairement de même forme que le préfixe nominal, ni selon l'élément sur lequel ils s'appliquent, mais l'allitération est assez fréquente, comme en attestent les exemples ci-dessous tirés de la langue kobiana.

Kobiana (langue atlantique, parlée en Guinée Bissau)

\begin{tabular}{|l|l|l|l|l|l|}
\hline (7) & a. & u-Yunaal & wo-ke & cet invité & classe sG, accord en classe sur le démonstratif \\
\hline & b. & i-Yunaal & $\mathbf{i}-\mathrm{ke}$ & ces invités & classe PL, accord en classe sur le démonstratif \\
\hline & c. & pu-fuur & $\mathbf{p u}-\mathrm{ke}$ & cette chambre & classe SG, accord en classe sur le démonstratif \\
\hline & d. & bu-lang & bu-ke & cette pirogue & classe sG, accord en classe sur le démonstratif \\
\hline & e. & & bu-r-aam & la mienne & classe sG, accord en classe sur le possessif \\
\hline & f. & ga-lang & ga-na & deux pirogues & classe PL, accord en classe sur le numéral \\
\hline
\end{tabular}

À la différence des classificateurs, les classes nominales sont obligatoires et ne dépendent pas de structures syntaxiques particulières. Les marques d'accord en classe apparaissent à l'intérieur des syntagmes nominaux pour indiquer l'accord entre le nom et ses dépendants, elles se retrouvent également ailleurs dans la phrase, dans le syntagme verbal (accord sujet et objet) par exemple.

\subsection{1 Évolution fonctionnelle des classificateurs}

L'évolution fonctionnelle des classificateurs, morphèmes rencontrés dans la majorité des langues d'Asie, sera illustrée par une comparaison entre deux dialectes sinitiques. Nous montrerons que dans les dialectes du Sud, les classificateurs apparaissent dans des contextes inédits dans les autres dialectes, remplissant de nouvelles fonctions en lien avec la spécification, une évolution dont l'explication se situe du côté du contact de langues.

Les exemples suivants illustrent une utilisation différente des classificateurs en chinois mandarin et en cantonais. Les deux langues font apparaitre des classificateurs dans les opérations de quantification et avec les démonstratifs (8). Cependant, seul le cantonais nécessite l'utilisation d'un classificateur comme morphème déterminant, un emploi de classificateur nominal (noun classifier) selon la typologie de Grinevald (2015: 813). Cet emploi, typique des langues de la péninsule indochinoise, est rare en mandarin ${ }^{3}$. Il est cependant fréquent dans les langues du sud de la Chine, mais aussi en thaï, en lao, en zhuang, en vietnamien, et dans les langues hmong (Vittrant et Mouton, à paraître). Cette zone géographique étant une aire linguistique (sprachbund) reconnue (Vittrant, 
2010), l'explication la plus probable à l'évolution fonctionnelle des classificateurs en Cantonais est celle du contact avec des langues du Sud-Est asiatique (Bisang, 1999 : 176).

Mandarin (d'après Bisang 1999 : 115, 145)

\begin{tabular}{|l|l|l|l|l|l|l|l|l|l|}
\hline (8) & a. & sān & fenng & xìn & b. & zhèi & fenng & xìn \\
\hline & & trois & CLF & lettre & & DEM & CLF & lettre \\
\hline & & \multicolumn{2}{|l}{ 'trois lettres' } & & \multicolumn{2}{|l}{ 'cette lettre' } \\
\hline
\end{tabular}

Cantonais (d'après Bisang 1999 : 148)

\begin{tabular}{|l|l|l|l|l|}
\hline & C. & ni1 & gan1 & fong4 \\
\hline & & DEM & CLF & pièce \\
\hline & & \multicolumn{3}{|c|}{ 'cette chambre/pièce' } \\
\hline
\end{tabular}

La comparaison des exemples (8b) et (9b) montre que le mandarin n'accepte pas la séquence CLF-N en position préverbale, tandis que le cantonais l'accepte. La séquence avec classificateur encode en cantonais le caractère spécifique (défini) du nom, une signification impossible en l'absence du classificateur (cf. 9a). Cette lecture spécifique sera rendue en mandarin par le nom nu (cf. 8a).

Mandarin (d'après Cheng \& Sybesma 2005)

\begin{tabular}{|l|l|l|l|l|l|}
\hline (9) & a. & gou & ai & chi & rou \\
\hline & & chien & aimer & manger & viande \\
\hline & & \multicolumn{2}{|c|}{ 'Le chien (spécifique)/Les chiens (en général) aime(nt) manger de la viande' } \\
\hline & b. & *zhi & gou & xihuan & chi \\
\hline & & CLF & chien & aimer.bien & manger \\
\hline & & \multicolumn{4}{|}{} \\
\hline
\end{tabular}

Cantonais (d'après Cheng \& Sybesma 2005)

\begin{tabular}{|l|l|l|l|l|l|l|}
\hline (10) & a. & gau & zhongyi & sihk & \multicolumn{1}{l|}{ juk } \\
\hline & & chien & aimer & manger & viande \\
\hline & & \multicolumn{5}{|c|}{ 'Le chien (spécifique) /Les chiens (en général) aime(nt) manger de la viande' } \\
\hline & b. & jek & gau & zhongyi & sihk & juk \\
\hline
\end{tabular}




\begin{tabular}{|l|l|l|l|l|l|}
\hline & CLF:animal & chien & aimer & manger & viande \\
\hline & \multicolumn{3}{|l}{ 'Le chien (spécifique) aime manger de la viande' } \\
\hline
\end{tabular}

\subsection{2 Évolution du système d'accord}

Le changement de fonction ou de valeur de morphèmes grammaticaux (réanalyse) peut être illustré par l'évolution du système d'accord des langues du groupe NYUN-BUY (Niger-Congo, Atlantique, Sénégal). Comme toutes les langues de la famille atlantique, les langues NYUN-BUY, forment le singulier et le pluriel de la plupart de leurs noms par la commutation de préfixes. Ces préfixes nominaux déclenchent par ailleurs un système d'accord dans différents éléments de la phrase. Dans les exemples ci-dessous de la langue kobiana, les noms baaz "baobab » et hak «main » déclenchent des accords de classes différents et marquent leur singulier et pluriel par des préfixes différents.

Kobiana (langue Niger-Congo, atlantique)

\begin{tabular}{|l|l|l|l|l|l|}
\hline (11) & u-baaz & u-kke & da-mbaaz & de-kke \\
\hline & u-baobab & ACCu-DEM2 & da-baobab & ACCde-DEM2 \\
\hline & \multicolumn{2}{|l|}{ 'Ce baobab' } & & 'Ces baobabs' \\
\hline$(12)$ & ja-hak & ji-heena & ya-hak & ya-na \\
\hline & ja-main & ACcji-un & ya-main & Accya-deux \\
\hline & 'Une main' & 'Deux mains' \\
\hline$(13)$ & bu-giis & bu-ke & ga-giis & ge-ke \\
\hline & bu-visage & Accbu-DEM2 & ga-visage & Accga-DEM2 \\
\hline & 'Ce visage' & 'Ces visages' \\
\hline
\end{tabular}

On dénombre en kobiana, sur ce principe, 27 appariements singulier / pluriel différents pour la part du lexique qui entre dans le système de classes (Voisin, 2015b).

En kobiana, à côté de ce système de classes, comme dans les autres langues de ce groupe, une part non négligeable des noms construit son équivalent pluriel à l'aide d'un suffixe et non par commutation des préfixes de classe (Voisin, 2015a). Ainsi dans l'exemple (14) de la langue kobiana, le nom pour 'jeune fille' baajid forme son pluriel par la suffixation du pluriel -a. Alors que dans les langues à classes, la formation du pluriel s'effectue normalement par la commutation de préfixe de classe singulier / pluriel comme vu dans les exemples (11), (12) et (13) précédents. Lorsque le pluriel est marqué par le suffixe, la valeur de singulier est non marquée, renvoyant à un système à deux valeurs dont seul le pluriel est marqué, comme attesté par exemple en anglais "box, boxes; child, children... ».

Kobiana (langue Niger-Congo, atlantique) (Voisin 2015b) 


\begin{tabular}{|l|l|l|l|l|}
\hline (14) & baajid & a-ke & baajid-a & ge-ke \\
\hline & jeune.fille & SG-DEM2 & jeune.fille-PL & PL-DEM2 \\
\hline & \multicolumn{2}{|l|}{ 'Cette jeune fille' } & 'Ces jeunes filles' \\
\hline
\end{tabular}

\subsection{Classification nominale et disparition}

Venons-en à la disparition d'items grammaticaux dans le domaine de la classification nominale. Nous l'illustrerons par l'évolution de l'usage des classificateurs dans une langue d'Asie orientale, et par les changements observés dans le système des classes nominales de langues atlantiques.

\subsubsection{Disparition dans l'usage des classificateurs}

Pamela Downing (1996), dans un ouvrage très complet sur le système des classificateurs en japonais, rend compte de la variété des usages des classificateurs en particulier selon l'âge des locuteurs. Au travers d'une enquête auprès de locuteurs et à travers un corpus de textes littéraires (romans, nouvelles, narrations), elle montre que la connaissance des formes classificatoires répertoriées par les grammaires est faible, et variable selon l'âge des locuteurs. Ainsi sur 154 formes qui remplissent les critères pour être catégorisées comme classificateur (p.17-18), seule une trentaine est régulièrement utilisée par les locuteurs à l'oral comme à l'écrit, avec une différence significative entre les locuteurs de moins de trente ans et les locuteurs plus âgés.

"[T]he mean number of forms used by adult speakers was only 136 for those over thirty, 28 for those under thirty. Even if my figures are somewhat exaggerated, though, they do indicate that speakers have at their disposal a body of classifiers considerably more numerous than the core group used frequently by all the speakers." (p.21)

Nous ajouterons pour finir la réflexion d'une jeune adulte (30 ans) locutrice de thaï interrogée dans le cadre d'une enquête sur les classificateurs, qui nous indiquait utiliser majoritairement un classificateur général (non-spécifié pour un trait sémantique) ...au grand dam de ses parents!

Thaï (Tai-kadai, Thaïlande)

\begin{tabular}{|c|c|c|c|c|c|c|c|c|c|}
\hline (15) & a. & $k^{\text {hăw }}$ & sú: & kānkrā & nùy & lêm & má:jbānt ${ }^{\text {hát }}$ & nùy & Pān \\
\hline & & $3 S G$ & acheter & ciseaux & un & CLF & règle & un & CLF \\
\hline & & lé? & rôm & nùy & $\mathbf{k}^{\mathbf{h}} \overline{\mathbf{a}} \mathbf{n}$ & & & & \\
\hline & & et & parapluie & un & CLF & & & & \\
\hline & & \multicolumn{8}{|c|}{ 'Il a acheté des ciseaux, une règle et un parapluie.' } \\
\hline & & & & & & & & & \\
\hline
\end{tabular}




\begin{tabular}{|c|c|c|c|c|c|c|c|c|c|}
\hline Loc. & b. & $\mathrm{k}^{\text {hăw }}$ & sú: & kānkrā & nùn & Pān & má:jbānt ${ }^{\mathrm{h}}$ át & nùy & Pān \\
\hline & & $3 \mathrm{SG}$ & acheter & ciseaux & un & CLF & règle & un & CLF \\
\hline & & lé? & rôm & nùy & Pān & & & & \\
\hline & & et & parapluie & un & CLF & & & & \\
\hline & & 'Il a & & & & & uie.' & & \\
\hline
\end{tabular}

\begin{tabular}{|c|c|c|c|c|c|c|c|c|c|}
\hline (16) & a. & $\mathrm{t}_{6} \mathrm{~h}$ ăn & mì: & dīnsǒ: & să:m & $t^{\text {h }} \hat{\varepsilon} \mathbf{y}$ & pà:kkā: & sì: & dâ:m \\
\hline & & $1 S G$ & avoir & crayon & trois & CLF & stylo & quatre & CLF \\
\hline & & lé? & jā:ylóp & sǒ:y & kô:n & & & & \\
\hline & & et & gomme & deux & CLF & & & & \\
\hline & & \multicolumn{8}{|c|}{ 'J'ai trois crayons, quatre stylos et deux gommes.' } \\
\hline \multirow[t]{4}{*}{ Loc. } & b. & t $6^{\text {hăn }}$ & mì: & dīnsǒ: & sǎ:m & Pān & pà:kkā: & sì: & Pān \\
\hline & & $1 S G$ & avoir & crayon & trois & CLF & stylo & quatre & CLF \\
\hline & & lé? & jā:ylóp & sつ̆:y & Pān & & & & \\
\hline & & et & gomme & deux & CLF & & & & \\
\hline & & 'J'ai tr & ayons, & re & set $c$ & $x$ gon & & & \\
\hline
\end{tabular}

Dans les exemples (15) et (16), l'énoncé (a) reflète un emploi traditionnel des noms lorsqu'ils sont quantifiés. Ils apparaissent avec les classificateurs renvoyant aux caractéristiques inhérentes des référents (lêm : CLF des objets longs et pointus ; ?ān : CLF des objets longs et plats; $\mathrm{k}^{\mathrm{h}} \overline{\mathrm{a}}$ : CLF des objets longs qui se manipulent avec la main; $\mathrm{t}^{\mathrm{h}} \hat{\varepsilon} y$ : CLF des objets longs, rigides et compacts ; dâ:m : CLF des objets longs, rigides noncompacts ; k̂̂:n : CLF des objets tridimensionnels de petite taille). Cet énoncé s'oppose à ceux fournis par notre locutrice en (b), lesquels montrent une utilisation réduite des classificateurs, avec l'emploi d'un seul classificateur peu spécifié sémantiquement (?ān) pour tous les référents quantifiés partageant la caractéristique générale d'être des référents plutôt longilignes. 


\subsubsection{Disparition de morphèmes de classes nominales}

Toutes les langues atlantiques ont un système de classes nominales. On considère même que le phylum Niger-Congo auquel ces langues sont reliées possédait déjà un système de classe pleinement grammaticalisé. Pour autant, toutes les langues du NigerCongo n'ont pas conservé ce système. Les langues ijoïdes, dogon, kru sont des familles de langues Niger-Congo qui dans l'ensemble ne conservent que quelques traces du système de classes originel. Dans les familles où ce système a été transmis, comme dans la famille atlantique, il est possible pour quelques langues de montrer que le système n'est plus clairement actif.

Dans les langues à système de classes plein, les lexèmes ont des préfixes qui marquent le nombre et ces préfixes nominaux sont liés à des morphèmes grammaticaux d'accord. Ces morphèmes entrent dans un système général de genre, et ces marques comme les marques de genre se retrouvent à différents niveaux syntaxiques pour donner des indications d'accord. Ainsi comme on peut le voir dans l'exemple ci-dessous du jóola keeraak, une langue atlantique parlée dans le Sud du Sénégal, les préfixes de classe bosur le verbe hay " dépasser » et $b$ - sur le connectif -ala marquent l'accord avec le nom tahęr « piqûre ».

Jóola keeraak (langue Niger-Congo, atlantique) (Segerer 2015)

\begin{tabular}{|c|c|c|c|}
\hline (17) & ba-tahecr-ab & b-ala & ha-łankIn-ah \\
\hline & ba-piqûre-DET.ACc.ba & ACc.ba-CONN & ha-abeille_sp-DET.Acc.ha \\
\hline
\end{tabular}

\begin{tabular}{|l|l|l|l|l|}
\hline bo-han- $\varepsilon$ & mə-sonji & b-ala & ha-aj-ah \\
\hline & Acc.ba-dépasser-ACP & ma-douleur & ACc.ba-conN & ha-abeille_sp-DET.ACC.ha \\
\hline & 'La piqûre des abeilles de mer fait plus mal que celle des abeilles de brousse.' \\
\hline
\end{tabular}

Dans la même famille de langues, le palor est une langue qui a perdu son système de classes. Les préfixes nominaux ont totalement disparu. Le marquage du nombre se fait bien au travers d'une commutation de forme en finale de mot. Ces marques sont très probablement diachroniquement issues d'un déterminant participant au système de classes. Dans le système actuel, on trouve une régularité pour la forme du pluriel avec la consonne $y$. Cependant, le singulier des noms est à apprendre, on dénombre 5 formes différentes possibles : $-v(\sim-f),-m,-k,-\varnothing$ et $-V$.

Palor (Diagne 2015)

\begin{tabular}{|l|l|l|l|}
\hline$(18)$ & $\mathrm{p} \varepsilon$ ? & $\mathrm{p} \varepsilon$ ?-f-a & $\mathrm{p} \varepsilon$ ?-y-a \\
\hline & 'une chèvre' & 'la chèvre' & 'les chèvres' \\
\hline$(19)$ & mIIs & mIIs-m-a & \\
\hline & 'du lait' & 'le lait' & \\
\hline
\end{tabular}




\begin{tabular}{|l|l|l|l|}
\hline$(20)$ & kilık & kIlık-k-a & kIlık-y-a \\
\hline & 'un arbre' & 'l'arbre' & 'les arbres' \\
\hline$(21)$ & pooni & pooni-v-a & pooni-y-a \\
\hline & 'un singe' & 'le singe' & 'les singes' \\
\hline
\end{tabular}

Comme on le voit dans l'exemple (22), ces marques de nombre ne déclenchent aucun système d'accord, aucune marque d'accord n'est identifiable sur les différents éléments de la phrase.

Palor (Diagne 2015)

\begin{tabular}{|l|l|l|l|l|l|}
\hline$(22)$ & ow-y-a & hom & kuncka & wor & wan \\
\hline & les_gens & s'asseoir & chacun & devoir & parler \\
\hline
\end{tabular}

\begin{tabular}{|l|l|l|l|l|l|l|}
\hline yecfo & wakda & jamma & min & lah & \\
\hline & qlq_chose & devoir & paix & pouvoir & avoir & \\
\hline & 'Dans une assemblée, chacun doit dire ce qu'il pense pour qu'il y ait la paix.' \\
\hline
\end{tabular}

La perte des préfixes sur les noms et la disparition du système d'accord se font de façon progressive. On peut voir à travers les exemples du wolof, le figement des préfixes nominaux dans certains noms. Dans la même langue, le système d'accord est encore présent dans le syntagme nominal (cf. exemples de (23) à (27)), mais il n'y a plus d'accord en classes sur le verbe (cf. exemples (28) et (29)).

Wolof

\begin{tabular}{|c|c|c|c|c|c|c|}
\hline (23) & jigéen & $\mathbf{j}-\mathbf{i}$ & jigéen & $j-u$ & jigéen & j-ii \\
\hline & femme & ACCj-DET.PROX & femme & ACCj-REL & femme & ACCj-DEM.PROX \\
\hline & \multicolumn{2}{|c|}{ 'la femme' } & \multicolumn{2}{|c|}{ 'une femme qui...' } & \multicolumn{2}{|c|}{ 'cette femme' } \\
\hline \multirow[t]{3}{*}{ (24) } & góor & $g-i$ & góor & g-u & góor & g-ii \\
\hline & homme & ACCg-DET.PROX & homme & ACCg-REL & homme & ACCg-DEM.PROX \\
\hline & \multicolumn{2}{|c|}{ 'l'homme' } & \multicolumn{2}{|c|}{ 'un homme qui...' } & \multicolumn{2}{|c|}{ 'cet homme' } \\
\hline \multirow[t]{3}{*}{ (25) } & bët & b-i & bët & b-u & bët & b-ii \\
\hline & œil & Accb-DET.PROX & œil & ACcb-REL & œil & Accb-DEM.PROX \\
\hline & \multicolumn{2}{|l|}{ 'l'œil' } & \multicolumn{2}{|c|}{ 'un œil qui...' } & \multicolumn{2}{|l|}{ 'cet œil' } \\
\hline
\end{tabular}




\begin{tabular}{|l|l|l|l|l|l|l|}
\hline (26) & wéñ & w-i & wéñ & w-u & wéñ & w-ii \\
\hline & mouche & ACCW-DET.PROX & mouche & ACCW-REL & mouche & ACCW-DEM.PROX \\
\hline & 'la mouche' & 'une mouche qui...' & 'cette mouche' \\
\hline$(27)$ & meew & m-i & meew & m-u & meew & m-ii \\
\hline & lait & Accm-DET.PROX & lait & Accm-REL & lait & ACcm-DEM.PROX \\
\hline & 'le lait' & 'du lait qui...' & 'ce lait' \\
\hline
\end{tabular}

\begin{tabular}{|l|l|l|l|l|}
\hline$(28)$ & jigéen & j-ii & rafet & na \\
\hline & femme & ACcj-DEM.PROx & être_belle & PARF.S.3SG \\
\hline & 'Cette femme est belle' & \\
\hline$(29)$ & bët & b-ii & rafet & na \\
\hline & cil & Accb-DEM.PROX & être_beau & PARF.S.3SG \\
\hline & 'Cet œil est beau' & \\
\hline
\end{tabular}

\subsection{Classification nominale et innovation}

\subsubsection{Les nouveaux classificateurs}

Les classificateurs, généralement d'origine nominale, forment une classe relativement ouverte. Il est ainsi possible de créer de nouveaux classificateurs en lien avec de nouvelles pratiques sociales.

Le dictionnaire de Nouveaux mots de l'Académie royale thailandaise (vol.2, 2009 :44) donne ainsi le morphème < dríy >, dérivé de l'anglais 'drink', comme un nouveau classificateur pour les boissons apéritives.

Thaï (Tai-Kadai, Thaïlande) (Vittrant \& Mouton à paraitre)

\begin{tabular}{|c|c|c|c|}
\hline \multirow[t]{2}{*}{ (30) } & a. & \multicolumn{2}{|c|}{ เครื่องดื่ม 1 ดริ๊งก์ } \\
\hline & $k^{h} r$ ûa:ydùu:m & nù̀y & drí (<ANG. DRINK) \\
\hline & boisson & un & CLF \\
\hline & \multicolumn{3}{|c|}{ 'un shot' (petit verre d'alcool) } \\
\hline
\end{tabular}

En Birmanie, l'apparition récente (dans le pays) de l'internet ainsi qu'un accès plus sûr au système bancaire a fait émerger le classificateur $/ \mathrm{kaJN}^{1} /$ dérivé de l'anglais 'account' qui s'emploie au côté du classificateur général $/ \mathrm{khu}^{1} /$, comme illustré en (31).

Birman (Tibéto-birman, Birmanie) -internet (blogspot $\left.{ }^{4}\right)$ 


\begin{tabular}{|c|c|c|c|c|c|c|c|c|c|}
\hline \multirow[t]{2}{*}{ (31) } & \multicolumn{8}{|c|}{ a. } & \multirow[b]{2}{*}{$=\mathrm{T} \varepsilon^{2}$} \\
\hline & khaiN $^{2} \mathrm{ma}^{2}$ & $n \varepsilon^{1}$ & facebook & tə & $\mathbf{k a O N}^{1}$ & $\mathrm{ya}^{1} \cdot \mathrm{fi}^{1}$ & $\theta w a^{3}$ & $=\mathrm{Pa}^{2}$ & \\
\hline & ê.ferme, solide & avec & facebook & un & CLF: compte & obtenir & ASP:perfect & POL & REAL \\
\hline & cla confirn & & 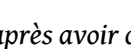 & & ́f], (on) ob & unc & te Faceboo & & \\
\hline
\end{tabular}

\begin{tabular}{|c|c|c|c|c|c|c|c|}
\hline \multirow[t]{2}{*}{ b. } & \multicolumn{7}{|c|}{ 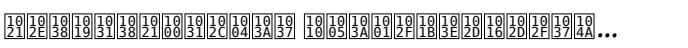 } \\
\hline & $P i^{3} m e^{3}$ & PəkaวN ${ }^{1}$ & tə & $\mathbf{k h u ^ { 1 }}$ & $\int \mathrm{i}^{1}$ & $=\mathrm{Pho}^{1}$ & $\ldots$ \\
\hline & email & compte & un & CLF: général & avoir & CONJ: $\mathrm{PO}$ & \\
\hline & \multicolumn{7}{|c|}{ 'Pour avoir un compte email...' } \\
\hline
\end{tabular}

\subsubsection{Innovation et classes nominales}

Par définition le système de classe est un système particulier d'accord. Nous avons vu dans les différents exemples illustrant ce système, que l'accord se fait sur plusieurs éléments de la phrase ; le cas le plus simple étant celui où le nom contrôleur de l'accord est présent. L'accord en classe peut se faire également en référence à un élément qui n'est pas dans la phrase, mais a déjà été présenté ou est sous-entendu. La marque d'accord joue alors le rôle d'indice pronominal et a une fonction anaphorique. Il existe également des emplois dits 'non contextuels'. Dans ce cas, le lien entre un éventuel contrôleur de l'accord et la marque d'accord peut s'expliquer par un phénomène d'ellipse. Ainsi, en l'absence de tous référents identifiables, même dans un contexte large, l'interprétation d'un pronom construit à partir de la consonne de classe l- en wolof renvoie par défaut à un inanimé. Dans l'exemple (32), la marque de classe l- est utilisée pour construire le relatif lu traduit par quelque chose. Littéralement, la construction wolof correspond approximativement à Il a y quelque chose qu'il sait sur les origines des Ndouts.

Wolof

\begin{tabular}{|l|l|l|l|}
\hline$(32)$ & Am & na & l-u \\
\hline & avoir & PARF.S.3SG & l-INDEF \\
\hline
\end{tabular}

\begin{tabular}{|l|l|l|l|l|l|l|}
\hline mu & xam & ci & cossanu & Ndutt & $y$-i \\
\hline & NARR.S.3SG & savoir & PREP & origine-CONN & ndout & y-DEF.PROX \\
\hline \multicolumn{7}{|l}{ 'Il sait quelque chose sur l'origine des Ndouts.' } \\
\hline
\end{tabular}

De la même façon, toujours en wolof, les consonnes de classe $k$ - pour le singulier et $\tilde{n}$ pour le pluriel renvoient par défaut aux êtres humains, comme on peut le voir dans les 
formes interrogatives $k$-an qui (SG) ? $\tilde{n}$-an qui (PL) ?. Les consonnes $k$ - et $\tilde{n}$-fonctionnent pour la $3^{\mathrm{e}}$ personne.

En plus de ces marques d'accord $k$ - et $\tilde{n}$ - en emploi non contextuel pour les êtres humains, la consonne de classe $m$ - est utilisée comme marque d'accord avec les noms propres (33) ou les pronoms (34).

Wolof

\begin{tabular}{|c|c|c|c|c|c|c|c|c|c|c|}
\hline \multirow[t]{2}{*}{ 33) } & \multicolumn{2}{|l|}{ Mel } & na & Makumba & \multicolumn{2}{|c|}{ m-ii } & toog & \multicolumn{2}{|r|}{ kasara } & g-i \\
\hline & \multicolumn{2}{|c|}{ ressembler } & PARF.S.3SG & Makumba & \multicolumn{2}{|c|}{ m-DEM.PROX } & asseo & \multicolumn{2}{|r|}{ bon_à_rien } & g-DEF.PROX \\
\hline & \multicolumn{9}{|c|}{ 'Il ressemble à Makumba celui qui est un bon à rien.' } & \\
\hline (34) & Man & \multicolumn{2}{|c|}{ m-ii } & di & wax & \multicolumn{4}{|l|}{$1-\mathrm{ii}, \ldots$} & \\
\hline 4) $\mathrm{M}$ & moi & \multicolumn{2}{|c|}{ m-DEM.PROX } & INACP & parler & \multicolumn{4}{|c|}{ 1-DEM.PROX } & \\
\hline
\end{tabular}

Cependant, cette affinité entre $k$-, ñ- et $m$ - et l'expression d'un référent humain ne tient pas au fait que l'ensemble des noms désignant les êtres humains entrent dans ces classes en wolof. Comme nous l'avons indiqué en introduction, le système de classes est un système grammaticalisé, ce qui implique entre autres, qu'il n'y a pas de motivation sémantique entre la classe et le référent. Les noms renvoyant à un être humain en wolof entrent dans des classes différentes et l'appariement $k$ - / $\tilde{n}$ - ne vaut que pour un seul nominal nit « être humain », comme on peut le voir sous (35).

Wolof

\begin{tabular}{|l|l|l|l|l|l|}
\hline (35) & jigéen & $\mathrm{j}-\mathrm{i}$ & jigéen & $\mathrm{y}-\mathrm{i}$ & 'la femme / les femmes' \\
\hline & ndaw & $\mathrm{s}-\mathrm{i}$ & ndaw & $\mathrm{y}-\mathrm{i}$ & 'la jeune fille / les jeunes filles' \\
\hline & coro & l-i & coro & $\mathrm{y}-\mathrm{i}$ & 'la petite amie / les petites amies' \\
\hline & far & $\mathrm{w}-\mathrm{i}$ & far & $\mathrm{y}-\mathrm{i}$ & 'le petit ami / les petits amis' \\
\hline & góor & $\mathrm{g}-\mathrm{i}$ & góor & $\mathrm{y}-\mathrm{i}$ & 'l'homme / les hommes' \\
\hline & xale & $\mathrm{b}-\mathrm{i}$ & $\mathrm{xale}$ & $\mathrm{y}-\mathrm{i}$ & 'l'enfant / les enfants' \\
\hline & morom & $\mathrm{m}-\mathrm{i}$ & morom & $\mathrm{y}-\mathrm{i}$ & 'le compagnon / les compagnons \\
\hline & nit & $\mathbf{k}-\mathrm{i}$ & nit & $\tilde{\mathbf{n}}-\mathrm{i}$ & 'l'être humain / les êtres humains' \\
\hline
\end{tabular}

À côté des utilisations non contextualisées des marques de classes, il y a également en wolof quelques consonnes dites de classes défectives qui renvoient à différentes notions générales telles que la localisation et la manière sans que cette notion puisse être associée à un nom référent dans la langue. Il s'agit des consonnes de classe $f$ - et $n$-. On 
ne peut les considérer comme marques d'accord, puisque aucun nom contrôleur n'existe dans la langue. Pour autant, ces consonnes se retrouvent dans les mêmes contextes que les autres marques d'accord, comme dans les interrogatifs par exemple $f$ an «où?", n-an «comment?" ou les indéfinis tels que f-eneen «ailleurs» et n-eneen « d'une autre manière ». De la même façon que nous avons vu précédemment que kan signifie qui ? (sG quelle personne ?) ñan qui ? (pL quelles personnes ?).

Il existe dans beaucoup de langues à classes un ensemble de marques de classes qui peuvent se poser sur une base dite "omniclasse » ou «base caméléon ». Le sens de ce mot est alors une interprétation par défaut de la classe et non de la base.

Kobiana

\begin{tabular}{|l|l|l|}
\hline (36) & base ro / ddo \\
\hline & u-ddo & arbre \\
\hline & de-ddo & arbres \\
\hline & a-ro & animal \\
\hline & ge-ro & animaux \\
\hline & ma-ro & liquide \\
\hline & gu-ro & objet \\
\hline & ña-ro & objets \\
\hline & pu-ddo & objet sans importance \\
\hline & pa-ddo & un seul objet, une seule graine, un fruit \\
\hline
\end{tabular}

Ces classes peuvent regrouper un nombre plus ou moins important de membres de la catégorie désignée par le terme générique ainsi créé. Ainsi en kobiana, il est vrai que la plupart des noms spécifiques d'arbres entrent dans l'appariement $u$ - / de- (37). En revanche, on ne peut faire le même constat pour les animaux: il n'est pas vrai par contre de dire que la plupart des animaux entrent dans l'appariement $a$ - / ge-. Seuls deux noms sont à l'heure actuelle répertoriés dans cet appariement ro/ddo « animal » et haka bras de mer, étendue d'eau salée ». Les noms d'animaux spécifiques sont répartis dans différents appariements (38) ou sont hors classe et prennent uniquement le suffixe -a pour marquer le pluriel (39).

\begin{tabular}{|l|l|l|l|}
\hline (37) & u-naana & da-naana & bananier / bananiers \\
\hline & u-mbaz & da-mbaz & baobab/baobabs \\
\hline$(38)$ & sa-kkun & na-kkun & scorpion / scorpions \\
\hline & u-ye & yu-ye & scolopendre / scolopendre \\
\hline
\end{tabular}




\begin{tabular}{|l|l|l|l|}
\hline (39) & muddum & muddum-a & hyène / hyènes \\
\hline & jamejj & jamajj-a & termite / termites \\
\hline
\end{tabular}

Dans cette dernière section, nous avons montré que l'innovation se rencontrait aussi dans les systèmes grammaticaux sous des formes variées: (1) modification du paradigme des classificateurs par l'ajout de nouvelles formes lesquelles reflètent une évolution des pratiques sociales dans les communautés, ou (2) extension des emplois des préfixes de classes vers des fonctionnements non-contextuels ou vers (re)sémantisation des préfixes nominaux dans le cas des « bases caméléons ».

\section{Conclusion}

Pour conclure, les changements mis en évidence pour le lexique dans les premières sections de cet article se retrouvent dans l'observation des changements possibles pour les items grammaticaux que sont les deux sous-systèmes de classification nominale étudiés. Ces changements mettent en jeu la réanalyse et ont des incidences sur le système grammatical ; ils conduisent parfois à identifier un profil typologique différent dans le cadre du marquage du nombre (3.1.2), par exemple. Les cas de disparition attestés au niveau lexical et au niveau grammatical sont connus depuis longtemps voir par exemple la perte du genre neutre dans le passage du latin au français, pour un exemple de langue plus proche. Ils existent aussi dans d'autres systèmes de classification nominale (3.2).

Les cas d'innovation sont plus étonnants au niveau grammatical. Mais comme le montrent les exemples des classificateurs (3.3.1), ils peuvent être motivés par des changements dans nos pratiques quotidiennes, de la même façon que pour l'innovation lexicale, et apparaître rapidement. On pourrait s'interroger sur les différences de temporalité dans ces changements en lien avec des critères tels que l'existence de système d'écriture et/ou d'instances de normalisation, de standardisation. Les langues qui servent d'illustration à cet article, relèvent de langues à tradition orale ou de langues à tradition écrite parfois très anciennes et aucune différence n'est notée. Comme l'atteste l'actualité récente, pour des langues avec des instances de normalisation fortes et un système d'écriture bien établi, l'innovation lexicale et les décisions sur le genre (classification nominale) sont rapides, voire instantanées. Ainsi, le terme coviD-19 a été dans les premiers temps utilisé au masculin. L'avis de l'Académie a imposé l'utilisation du féminin, lequel s'est imposé a minima dans les médias et les discours officiels. Dans les usages, le masculin reste fortement présent et dans les constructions syntaxiques telles que "la pandémie de », le masculin est utilisé par tous, «la pandémie du coviD-19 », « *la pandémie de la coviD-19 ».

Abréviations

Accbu $=$ accord de classe nominale bu- ; bu= préfixe nominal de classe bu- ; Accga= accord de classe nominale ga-; $A S P=$ aspect; ga $=$ préfixe nominal de classe ga-; $\mathrm{CLF}=$ classificateur ; CONJ= conjonction ; $\mathrm{DEM} 2=$ démonstratif 2 ; $\mathrm{NEG}=$ négation ; PL= pluriel ; POL= politesse ; REAL= realis ; $\mathrm{SG}=$ singulier ; 


\section{BIBLIOGRAPHIE}

Anward, Jan, Edith Moravcsik, et Leon Stassen. 1997. « Parts of speech: A challenge for typology ». Linguistic Typology 1 (2): 167-83.

Bisang, Walter. 1999. «Classifiers in East and Southeast Asian Languages: Counting and Beyond ». In Numeral types and changes worldwide, par J. Gvozdanovic, 113-86. Muchen: Walter de Gruyter.

Boltanski, Jean-Élie. 1998. La linguistique diachronique. Que sais-je? Paris: Presses Universitaires de France - PUF.

Cheng, Lisa Lai-Shen, et Rint Sybesma. 2005. «Classifiers in four varieties of Chinese ». In Handbook of Comparative Syntax, par Guglielmo Cinque et Richard S. Kayne, 259-92. Oxford: Oxford University Press.

Corbett, Greville G. 1991. Gender. Cambridge: Cambridge University Press.

Creissels, Denis. 1991. Description des langues négro-africaines et théorie syntaxique. Grenoble: ELLUG.

Creissels, Denis, et Konstantin Pozdniakov, éd. 2015. Les classes nominales dans les langues atlantiques. Grammatical Analyses of African Languages 49. Köln: Rudiger Köppe Verlag.

Diagne, Anna M. 2015. « Le palor et le ndut: deux langues atlantiques sans classes nominales ». In Les classes nominales en laalaa, par Konstantin Pozdniakov et Denis Creissels, 261-90. Köln: Rüdiger Köppe Verlag.

Downing, Pamela. 1996. Numeral Classifier Systems: The Case of Japanese. Amsterdam: John Benjamins.

Fedden, Sebastian, et Greville G. Corbett. 2018. « Extreme classification ». Édité par De Gruyter Mouton. Cognitive Linguistics 29 (4): 633-75.

Feuillet, Jack. 1983. « Se débarrassera-t-on un jour des parties du discours? » Bulletin de la Société de Linguistique de Paris 78 (1): 23-51.

Givón, Talmy. 1971. « Historical syntax and synchronic morphology: an archeologist's field trip ». Papers from the 7th Meeting of the Chicago Linguistic Society. Chicago: The Chicago Linguistic Society.

Grinevald, Colette. 1999. « Typologie des systèmes de classification nominale ». Faits de langues 7 (14): 101-22.

Grinevald, Colette. 2015. « Classifiers, Linguistics of ». In International Encyclopedia of the Social \& Behavioral Sciences (Second Edition), édité par James D. Wright, Second Edition, 811-18. Oxford: Elsevier.

Heine, Bernd. 1984. Grammaticalization and Reanalysis in African Languages. Édité par Mechthild Reh. Hamburg: H. Buske.

Heine, Bernd. 1993. Auxiliaries. Cognitive Forces and Grammaticalization. New York: Oxford Univ. Press.

Heine, Bernd. 1997. Possession: Cognitive Sources, Forces, and Grammaticalization. Cambridge: Cambridge University Press.

Heine, Bernd. 2003. « Grammaticalization ». In The Handbook of Historical Linguistics, par Brian D. Joseph et Richard D. Janda, 575-601. Blackwell Handbooks in Linguistics. Malden, MA: Blackwell Pub. 
Heine, Bernd, et Tania Kuteva. 2005. Language Contact and Grammatical Change. Cambridge Approaches to Language Contact. Cambridge New York (N.Y.): Cambridge University Press.

Heine, Bernd, et Tania Kuteva. 2008. « Constraints on Contact-Induced Linguistic Change ». Journal of Language Contact 2 (1): 57-90.

Heine, Bernd, et Tania Kuteva. 2010. « Contact and grammaticalization ». In The handbook of language contact, par Raymond Hickey, 86-105. Blackwell handbooks in linguistics. Chichester, West Sussex ; Malden, MA: Wiley-Blackwell.

Iwasaki, Shoichi, et Preeya Ingkaphirom. 2005. A Reference Grammar of Thai. Cambridge: Cambridge University Press.

Laforest, Serge. 1972. Gaunce se pique au jeu. Paris: Fleuve noir.

Marchello-Nizia, Christiane. 1999. Le français en diachronie : douze siècles d'évolution. Paris-Gap: Ophrys.

Marchello-Nizia, Christiane. 2006. Grammaticalisation et changement linguistique. Champs linguistiques. Bruxelles: De Boeck/Duculot.

Meillet, Antoine. 1912. «L'évolution des formes grammaticales ». In Linguistique historique et linguistique générale, par Antoine Meillet, Société de Linguistique de Paris, 1:131-48. Linguistique, VIII. Paris: Champion.

Peyraube, Alain. 2002. « L'évolution des structures grammaticales. » Langages 36 (146): 46-58.

Pozdniakov, Konstantin. 2016. «Tendances actuelles en comparatisme et reconstruction (présentation générale) ». Faits de langues 47: 5-24.

Rey, Alain. 2013. Le voyage des mots : De l'Orient arabe et persan vers la langue française. Paris: Guy Trédaniel éditeur.

Robert, Stéphane. 2005. « The challenge of polygrammaticalization for linguistic theory: fractal grammar and transcategorial functioning ». In Linguistic Diversity and Language Theories, par Zygmunt Frajzyngier, Adam Hodges, et David S. Rood, 119-42. Studies in language companion series 72. Amsterdam: John Benjamins.

Saussure, Ferdinand de. 1916. Cours de linguistique générale. [Réed. 1972]. Paris: Payot.

Segerer, Guillaume. 2015. « Les classes nominales en keerak (joola de Kabrousse) ». In Les classes nominales dans les langues atlantiques, par Denis Creissels et Konstantin Pozdniakov, 103-46. Grammatical Analyses of African Languages 49. Köln: Rudiger Köppe Verlag.

Thomason, Sarah Grey, et Terrence Kaufman. 1988. Language Contact, Creolization, and Genetic Linguistics. Berkeley, Los Angeles: University of California Press.

Tuite, Kevin. 1999. « Au-delà du Stammbaum: Théories modernes du changement linguistique ». Anthropologie et Sociétés 23 (3): 15.

Vittrant, Alice. 2005. «Classifier systems in Burmese ». In Proceedings of Twenty-Eighth Annual Meeting of Berkeley Linguistic Society, édité par Patrick Chew, 129-48. Berkeley, CA.

Vittrant, Alice. 2010. « Aire linguistique Asie du Sud-Est continentale : le birman en fait-il partie? » Moussons 16: 7-38.

Vittrant, Alice. 2019. « Burmese ». In The Mainland Southeast Asia Linguistic Area, édité par Alice Vittrant et J Watkins, 56-130. Berlin ; Boston: De Gruyter Mouton. 
Vittrant, Alice, et Léa Mouton. à paraître. « La classification nominale dans les langues d'Asie du Sud-est ». Édité par Nicolas Quint et Sylvie Voisin. Faits de Langues Classification nominale : nouvelles approches, nouvelles données, nouveaux enjeux.

Vittrant, Alice, et Marc Tang. à paraître. «Classifiers in Southeast Asian Languages ». In The Languages and Linguistics of Mainland Southeast Asia: a comprehensive guide, par M. Jenny et P. Sidwell. Berlin: Mouton de Gruyter.

Voisin, Sylvie. 2015a. « Sur l'origine du suffixe du pluriel dans le groupe nyun-buy ». LambertLucas, Linguistique et Langues Africaines, 1: 13-41.

Voisin, Sylvie. 2015b. « Les classes nominales en kobiana ». In Les classes nominales dans les langues atlantiques, par Denis Creissels et Konstantin Pozdniakov, 324-80. Grammatical Analyses of African Languages 49. Köln: Rudiger Köppe Verlag.

Welmers, William E. 1973. African Language Structures. Berkeley, Los Angeles, London: University of California Press.

Yaguello, Marina. 1998. Petits faits de langues. Paris: Seuil.

\section{NOTES}

1. https://orthogrenoble.net/mots-nouveaux-dictionnaires/sorties-petit-larousse-1998/

2. Les dates renvoient aux $1^{\text {ère }}$ et $8^{\mathrm{e}}$ éditions du dictionnaire de l'Académie française accessible en ligne (https://www.dictionnaire-academie.fr)

3. La séquence CLF-N en mandarin, a toujours une lecture indéfinie et une position objet (Cheng et Sybesma 2005), est souvent analysée comme la réduction phonologique de la séquence CLF-N avec le numéral 'un', soit 'yi-CLF-N'.

4. Voici le blog dont sont extraits les exemples : http://knowladgeshar.blogspot.com/

\section{RÉSUMÉS}

Dans cet article, nous montrerons que les items grammaticaux suivent les mêmes processus de changement que les items lexicaux à travers l'observation de deux systèmes de classification nominale. Nous illustrons dans un premier temps les changements observables dans le lexique, que ces changements soient dus à la disparition d'une forme ou d'une fonction, ou à une innovation par apparition ou réanalyse d'une forme. Dans un deuxième temps, nous montrons que les outils grammaticaux changent et que ces transformations, sans pleinement modifier le système, impliquent des réajustements qui peuvent passer relativement inaperçus, comme dans le cas des réanalyses lexicales, mais n'en affectent pas moins profondément le système. Les deux systèmes de classification nominale qui sont abordés ont la particularité d'être aux extrêmes d'une échelle de grammaticalité (Fedden \& Corbett 2018). Les classificateurs constituent un système lexico-grammatical de classification des noms assez motivé, très présent dans les langues d'Asie. À l'inverse, les classes nominales sont des systèmes beaucoup plus arbitraires et considérés comme fortement grammaticalisés. Ils sont largement présents dans les langues Niger-Congo. Il est possible de montrer que, dans les deux types de systèmes, les morphèmes 
grammaticaux peuvent changer de fonction ou de valeur, que ce constat résulte d'une comparaison avec d'autres langues (apparentées) ou d'une perte des propriétés attendues. Nous montrons également que les morphèmes grammaticaux peuvent s'étioler ou disparaître complètement ou qu'ils peuvent être le résultat d'innovations récentes. Notre article accrédite ainsi l'idée que les changements linguistiques identifiés pour le lexique, le sont pour l'ensemble des éléments qui composent le système d'une langue, des sons aux lexèmes en passant par les morphèmes grammaticaux.

In this paper, we show that grammatical items follow the same processes of change as lexical items through the observation of two nominal classification systems. We first illustrate changes occurring in the lexicon, whether these changes are due to the disappearance of a form or a function, or to an innovation by the appearance or reanalysis of a form. Secondly, we show that grammatical tools also change and that these changes, without fully modifying the system, involve readjustments which can go relatively unnoticed - as in the case of lexical reanalyses -, but nonetheless have a profound effect on the system. The two nominal classification systems discussed are located at both ends of a grammaticality scale (Fedden \& Corbett 2018). Classifiers constitute a fairly motivated lexico-grammatical system for classifying names, very common in Asian languages. Conversely, nominal classes are much more arbitrary systems and considered to be highly grammaticalized. They are widely present in the Niger-Congo languages. In both types of systems, grammatical morphemes can change function or value, whether this observation results from a comparison with other (related) languages or from a loss of the expected properties. We also show that grammatical morphemes can fade away or disappear altogether. They can also be the result of recent innovations. Our article thus supports the idea that the linguistic changes identified for the lexicon are identified for all elements that make up the system of a language, from sounds to lexemes through to grammatical morphemes.

\section{AUTEURS}

\section{ALICE VITTRANT}

Université Lyon 2 / CNRS-DDL UMR 5596

SYLVIE VOISIN

Aix Marseille Université / CNRS-DDL UMR 5596 